\title{
Interactions of ataxin-3 with its molecular partners in the protein machinery that sorts protein aggregates to the aggresome
}

\author{
Marcella Bonanomi ${ }^{a}$, Serena Mazzucchelli ${ }^{a}$, Annalisa D’Urzo ${ }^{a}$, Marco Nardini $^{\mathrm{c}}$, \\ Petr V. Konarev ${ }^{b}$, Gaetano Invernizzi ${ }^{a}$, Dmitri I. Svergun ${ }^{b}$, Marco Vanoni ${ }^{a, d}$, \\ Maria Elena Regonesi ${ }^{\mathrm{a}, *}$, Paolo Tortora ${ }^{\mathrm{a}}$ \\ a Department of Biotechnologies and Biosciences, University of Milano-Bicocca, P.zza della Scienza 2, 20126 Milan, Italy

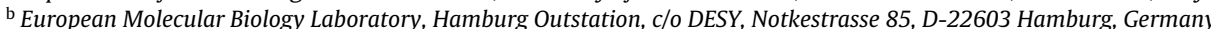 \\ ${ }^{c}$ Department of Biosciences, University of Milan, Via Celoria 26, 20133 Milano, Italy \\ d SysBio Centre for Systems Biology, Milan and Rome, Italy
}

\section{A R T I C L E I N F O}

\section{Article history:}

Received 14 October 2013

Received in revised form 5 March 2014

Accepted 20 March 2014

Available online 28 March 2014

\section{Keywords:}

Ataxin-3

HDAC6

Tubulin dimer

Small angle X-ray scattering

Surface plasmon resonance

\begin{abstract}
A B S T R A C T
Ataxin-3 (AT3) is the protein that triggers the inherited neurodegenerative disorder spinocerebellar ataxia type 3 when its polyglutamine (polyQ) stretch close to the C-terminus exceeds a critical length. AT3 consists of the N-terminal globular Josephin domain (JD) and the C-terminal disordered one. It cleaves isopeptide bonds between ubiquitin monomers, an event involved in protein quality control mechanisms. AT3 has been implicated in the pathway that sorts aggregated protein to aggresomes via microtubules, in which dynein and histone deacetylase 6 (HDAC6) also seem to be involved. By taking advantage of small angle X-ray scattering (SAXS) and surface plasmon resonance (SPR), we have investigated the interaction of AT3 with tubulin and HDAC6. Based on SAXS results, the AT3 oligomer, consisting of 6-7 subunits, tightly binds to the tubulin hexameric oligomer in a "parallel" fashion. By SPR analysis we have demonstrated that AT3 binds to tubulin dimer with a $50 \mathrm{nM}$ affinity. Binding fits with a Langmuir 1:1 model and involves a single binding interface. Nevertheless, the interaction surface consists of three distinct, discontinuous tubulin-binding regions (TBR), one located in the JD, and the two others in the disordered domain, upstream and downstream of the polyQ stretch. In the absence of any of the three TBRs, the affinity is drastically reduced. By SPR we have also provided the first evidence of direct binding of AT3 to HDAC6, with affinity in the range $0.1-1 \mu \mathrm{M}$. These results shed light on the interactions among the components of the transport machinery that sorts aggregate protein to the aggresome, and pave the way to in vivo studies aimed at further clarifying their roles.
\end{abstract}

(C) 2014 Elsevier Ltd. All rights reserved.

\section{Introduction}

Polyglutamine (polyQ) expansion diseases are hereditary neurodegenerative disorders that develop when a CAG repeat present in the respective encoding gene is expanded above a certain threshold, mostly 40-50 consecutive residues. The expansion results in misfolding and other structural rearrangements in the protein product, which lead to aberrant interactions of the expanded protein and to the consequent formation of fibrillar amyloid-like aggregates (Zoghbi and Orr, 2000; Gatchel and Zoghbi, 2005). The

* Corresponding author at: P.zza della Scienza 2, 10126 Milano, Italy. Tel.: +39 02 64483437; fax: +390264483565.

E-mail address: mariaelena.regonesi@unimib.it (M.E. Regonesi). loss of function resulting from misfolding might also be involved in the pathogenesis mechanisms (Zuccato et al., 2001, 2003).

Machado-Joseph disease is the most common form of autosomal dominantly inherited ataxia and its causative agent is the polyQ protein ataxin-3 (AT3). AT3 is composed by a structured globular N-terminal domain, the Josephin domain (JD), followed by a flexible C-terminal tail containing the polyQ stretch (Burnett et al., 2003; Masino et al., 2003). Different AT3 isoforms resulting from alternative splicing have been described; the most common in the human brain has three ubiquitin-interacting motifs (UIMs) in the disordered domain, one located downstream of the polyQ sequence (Harris et al., 2010). Even though its physiological role is not yet fully understood, it has been established that AT3 is a cysteine protease capable of cleaving isopeptide bonds between ubiquitin monomers (Burnett et al., 2003), an event involved in the protein quality control mechanisms (Doss-Pepe et al., 2003). 
It is also clear that AT3's capability of binding polyubiquitylated proteins via its UIMs is important to position polyubiquitylated chains for the subsequent cleavage (Winborn et al., 2008; Chai et al., 2004). Moreover, AT3 seems to play a role in the aggresome pathway. Aggresomes are perinuclear proteinaceous aggregates, close to the microtubule-organizing center (MTOC), to which misfolded protein is sorted via microtubules when proteasomes are overloaded or their function compromised (Garcia-Mata et al., 1999). In particular, it is known that AT3 co-localizes with aggresome and preaggresome particles and co-precipitates with dynein and histone deacetylase 6 (HDAC6) (Burnett and Pittman, 2005). HDAC6 plays a pivotal role in the formation of aggresomes: in particular, the ubiquitin-binding activity of HDAC6 has been shown to mediate the transport of polyubiquitylated proteins along microtubule tracks to aggresomes (Kawaguchi et al., 2003). Recently, it has been demonstrated that HDAC6 binds polyubiquitylated proteins through the unanchored C-terminal diglycine motif of ubiquitin that are likely to be released by the deubiquitinating activity of AT3 (Ouyang et al., 2012). Furthermore, in a previous work we demonstrated that AT3 tightly binds tubulin dimers, the constituents of microtubules (Mazzucchelli et al., 2009). Microtubules are essential components of the cytoskeleton that play a major role in many cellular functions, including the retrograde transport of misfolded proteins to the aggresomes at MTOC (de Forges et al., 2012).

Here, we present investigations aimed at providing further insight into the mode of interaction of AT3 with the protein components that are involved in sorting aggregated proteins to the aggresome. In particular, by taking advantage of small angle X-ray scattering (SAXS) and surface plasmon resonance (SPR) methods we could model the scaffold of the AT3-tubulin complex. SAXS data are consistent with the indication that the JD units contact the tubulin surface at the region that would be external in a tubulin protofilament. SPR measurements identify in AT3 three separate tubulin interacting regions (TBR), one within JD and the two others within the unstructured region. SPR experiments also provide the first evidence of a direct binding of AT3 to HDAC6. Our findings pave the way to a better understanding of the role of the individual components of the machinery that sorts proteins to the aggresome.

\section{Experimental procedures}

\subsection{Cloning and expression of AT3 variants}

The truncated forms $\mathrm{AT}_{1-182}$ and $\mathrm{AT}_{1-291}$ were cloned in fusion with glutathione S-transferase in a pGEX-6P-1 (GE Healthcare LifeSciences, Little Chalfont, UK) as previously described (Mazzucchelli et al., 2009). The truncated variants AT3 ${ }_{182-362}$, $\mathrm{AT}_{1-244}$, AT3 ${ }_{1-319}$ were obtained by PCR reactions using $5^{\prime}$ phosphorylated oligos and the construct pGEX6P1/AT3Q24 as a template. These AT3 variants were expressed in E. coli strain BL21CodonPlus (DE3)-RIL (E. coli B F- ompT hsdS (rB- mB-) dcm+ Tetr gal $\lambda$ (DE3) endA Hte [argU ileY leuW Cam ${ }^{r}$ ] (Stratagene, La Jolla, CA, USA) as GST-fusion proteins containing a PreScission Protease recognition site. The wild type AT3Q24 was cloned in a PQE30 vector (Qiagen Hamburg GmbH, Hamburg, Germany) and expressed as a His-tagged protein as previously described (Natalello et al., 2011). The variants AT3Q6 and AT3Q24-3UIM were obtained by PCR reactions using 5'-phosphorylated oligos and the construct pQE30/AT3Q24 as template. These three variants were expressed in SG13009 (E. coli K12 $\mathrm{Nal}^{\mathrm{S}}, \mathrm{Str}^{\mathrm{S}}, \mathrm{Rif}^{\mathrm{S}}$, $\mathrm{Thi}^{-}, \mathrm{Lac}^{-}, \mathrm{Ara}^{+}, \mathrm{Gal}^{+}, \mathrm{Mtl}^{-}, \mathrm{F}^{-}$, $\mathrm{RecA}^{+}, \mathrm{Uvr}^{+}$, Lon $^{+}$; Qiagen Hamburg GmbH, Hamburg, Germany) as His-tagged proteins.

\subsection{Purification of AT3 variants}

The procedures adopted to purify either GST- or His-tagged AT3 variants shared the initial steps, the only difference being that for the GST-tagged construct (i.e. AT3Q24 and AT3 $182-362$ ), cells were grown at $37^{\circ} \mathrm{C}$ in LB-ampicillin medium, for the His-tagged in LB-ampicillin-kanamycin medium. In either case, they were induced with $50 \mu \mathrm{M}$ IPTG at $A_{600} 0.8$ for $3 \mathrm{~h}$ at $30^{\circ} \mathrm{C}$. GST-tagged proteins were purified by Glutathione Sepharose 4 Fast Flow affinity chromatography. Except for AT3/JD $\Delta$, GST was then removed proteolytically using Prescission Protease. When purifying the Histagged variants, the procedure by Chow et al. (2006) was followed with minor modifications. Before each experiment, purified protein preparations were thawed, centrifuged at $15,000 \times \mathrm{g}$ for $15 \mathrm{~min}$ at $4{ }^{\circ} \mathrm{C}$ to remove aggregates and then equilibrated with PBS buffer (for further details see Supplementary Methods). Protein content was determined using Coomassie brilliant blue G-250 (Thermo Fisher Scientific, Rockford, IL, USA) and BSA as a standard protein. To ensure that the protein variants under investigation were properly folded, they were routinely subjected to FT-IR analysis to assess secondary structure content (Natalello et al., 2011).

\subsection{Small-angle X-ray scattering (SAXS)}

SAXS patterns of AT3Q24, tubulin and AT3Q24-tubulin complexes in PBS buffer were recorded at X33 EMBL beamline on the storage ring DORIS-III (Hamburg, Germany) (Roessle et al., 2007). The protein concentration of the measured samples ranged between 2 and $10 \mathrm{mg} / \mathrm{ml}$. The data were recorded at $10^{\circ} \mathrm{C}$ using pixel 1M PILATUS detector (DECTRIS, Switzerland) (X33) at a sample-detector distance of $2.7 \mathrm{~m}$, respectively, and a wavelength of $\lambda=0.15 \mathrm{~nm}$, covering the range of momentum transfer $0.12 \mathrm{~nm}^{-1}<s<5.5 \mathrm{~nm}^{-1}(s=4 \pi \sin \theta / \lambda$, where $2 \theta$ is the scattering angle). No measurable radiation damage was detected by comparison of four successive time frames with $30 \mathrm{~s}$ exposures. The data were averaged after normalization to the intensity of the incident beam, corrected for the detector response, and the scattering of the buffer was subtracted. All data manipulations were performed by using the program package PRIMUS (Konarev et al., 2003). The program DAMMIF (Franke and Svergun, 2009), a fast version of DAMMIN (Svergun, 1999), was used to reconstruct the low resolution shape of AT3Q24, tubulin and AT3Q24-tubulin complexes. The results of multiple DAMMIF runs (20 runs) were averaged to determine common structural features using the programs DAMAVER (Volkov and Svergun, 2003) and SUPCOMB (Kozin and Svergun, 2001). The aggregation states of the AT3Q24 and tubulin proteins were estimated from their excluded (Porod, 1982) volumes taking into account that for sufficiently large globular proteins the hydrated volume in $\mathrm{nm}^{3}$ should numerically be about twice the molecular mass in kDa. Molecular modeling for the AT3Q24-tubulin complex was done using the NMR atomic model of the JD of AT3Q24 (PDB code; 1YZB [Nicastro et al., 2005]) and the crystallographic model of tubulin dimer (PDB code: 1TUB and 1SA0 [Nogales et al., 1998; Ravelli et al., 2004]) by manual docking to the $a b$ initio model of the complex. The scattering pattern from the constructed model was calculated from its atomic coordinates by the program CRYSOL (Svergun et al., 1995) (see Supplementary Methods).

\subsection{Surface plasmon resonance (SPR)}

A BIACORE X system (GE Healthcare Life Sciences, Little Chalfont, England) was used to analyze molecular interactions by means of SPR. Tubulin (dimer) and HDAC6 proteins were coupled to a carboxymethylated dextran surface of two different CM5 sensor chips by using amine-coupling chemistry at surface densities of 4000 and 3500 resonance units, respectively. Appropriate, multiple concentrations of the interacting proteins (analytes in BIAcore terminology) were injected at $25^{\circ} \mathrm{C}(30 \mu \mathrm{l}$ injections at a flow rate of $10 \mu \mathrm{l} / \mathrm{min}$ ) in running buffer (10 mM HEPES, pH 7.4, $150 \mathrm{mM} \mathrm{NaCl}$, 
3 mM EDTA containing $0.005 \%$ (v/v) Surfactant P20). After injection, analyte solutions were replaced by running buffer at a continuous flow rate of $10 \mu \mathrm{l} / \mathrm{min}$. Surface regeneration was accomplished by injecting $50 \mathrm{mM} \mathrm{NaOH}(10 \mu \mathrm{l} / \mathrm{min} ; 0.5$-min contact time). Each sensorgram was subtracted for the response observed in the control flow cell (no immobilized protein) and normalized to a baseline of 0 $\mathrm{RU}$. The interaction rate constants were calculated by using the BIA evaluation 4.1 SPR kinetic software (GE Healthcare Life Sciences, Little Chalfont, England).

\section{Results}

\subsection{SAXS analysis of the tubulin-ataxin-3 complex}

Previous findings have shown that ataxin-3 (AT3) is part of the transport machinery of misfolded proteins to the aggresome via microtubules (Burnett and Pittman, 2005). We showed, in addition, tight binding of AT3 (dissociation constant of 50-70 nM) to tubulin dimers (Mazzucchelli et al., 2009). Here we aim at providing a better understanding of such mode of binding, and consequently of the molecular mechanism by which AT3 fulfills such physiological role.

To obtain a structural description of the AT3Q24-tubulin complex, we resorted to a strategy based on SAXS measurements and molecular modeling validated by experimental data. We selected the conditions whereby both proteins are less prone to oligomerize and acquired and compared SAXS data for each of the isolated individual components, as well as for the complex.
The experimental curve for tubulin is shown in Fig. 1A, upper panel. The estimated molecular weight of the solute $\left(\mathrm{MW}_{\text {exp }}=280 \pm 20 \mathrm{kDa}\right)$ indicates that the protein is composed of three $\alpha \beta$-dimeric units (each monomer having a theoretical MW $\sim 55 \mathrm{kDa}$ ) under the assayed experimental conditions. Accordingly, the excluded volume of the particle in solution (Porod volume) is $V_{p}=450 \pm 20 \times 10^{3} \AA^{3}$, noting that for globular proteins the hydrated volume in $\AA^{3}$ should numerically be about twice the molecular mass in Da. The experimental radius of gyration $R_{g}$ and maximum size $D_{\max }(7.0 \pm 0.3 \mathrm{~nm}$ and $25 \pm 1 \mathrm{~nm}$, respectively) point to an elongated particle structure. The low resolution shape of hexameric tubulin, reconstructed ab initio using DAMMIN (Svergun, 1999), has the overall size of about $40 \AA \times 60 \AA \times 250 \AA$, fitting the experimental data with discrepancy $\chi=1.05$ (Fig. $1 \mathrm{~A}$ ). High resolution modeling of the tubulin oligomer within the low resolution $a b$ initio shape resulted in a linear combination of three $\alpha \beta$-dimers similar to that seen in the tubulin protofilament (PDBcode 1TUB) (Nogales et al., 1998, 1999). The oligomer is not fully straight, with the terminal dimer slightly bent relative to the axis of the preceding linear tetramer (Fig. 1A, lower panel), in a conformation reminiscent of that found in the crystal structure of tubulin in complex with the stathmin-like domain of RB3 and colchicine (PDB-code 1SA0) (Ravelli et al., 2004). The SAXS curve computed from our hexameric model yielded a good fit to the experimental data with $\chi=1.09$.

AT3Q24 consists of the Josephin domain (JD; about $20 \mathrm{kDa}$ : NMR structure PDB-code 1YZB) and a $20 \mathrm{kDa}$ unstructured region (Nicastro et al., 2005). Fig. 1B, upper panel shows the

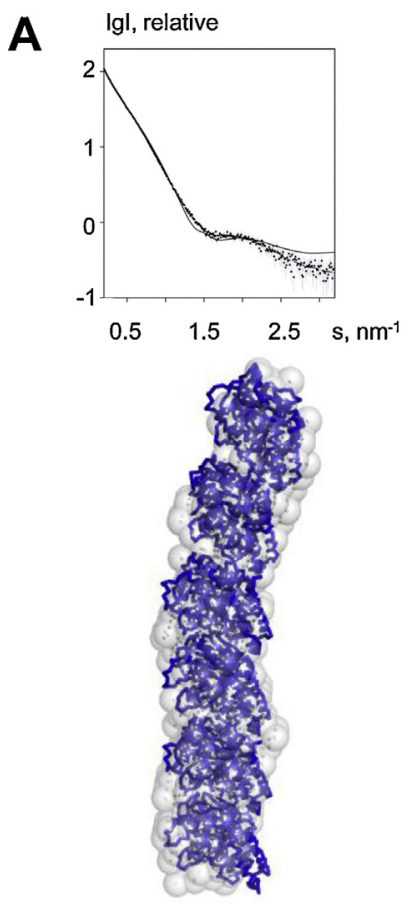

tubulin
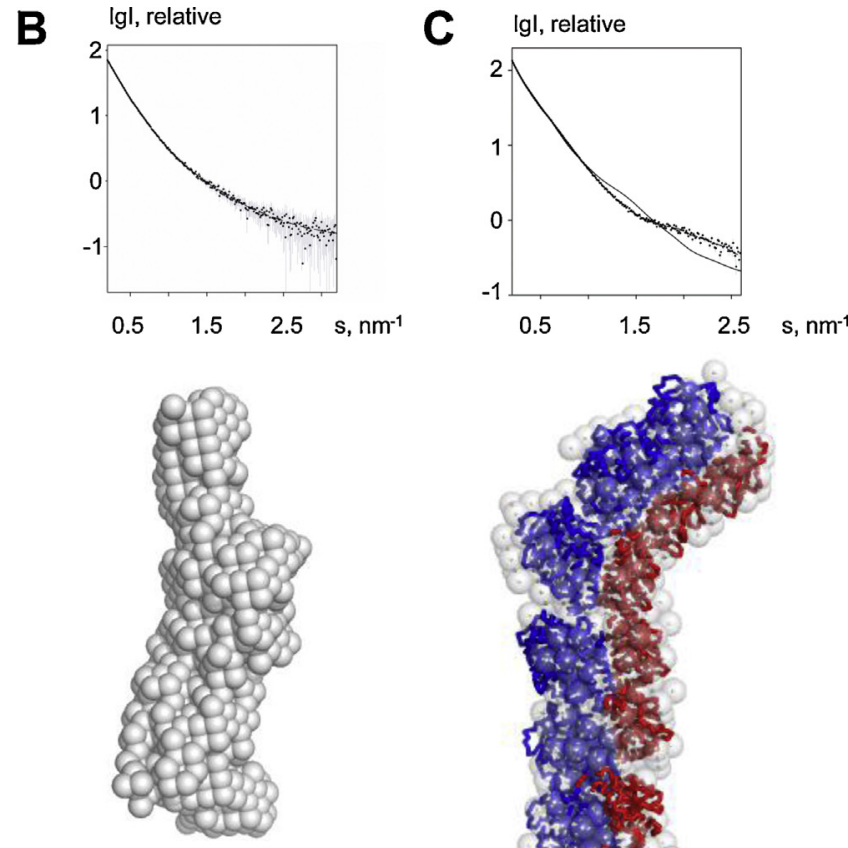

AT3Q24

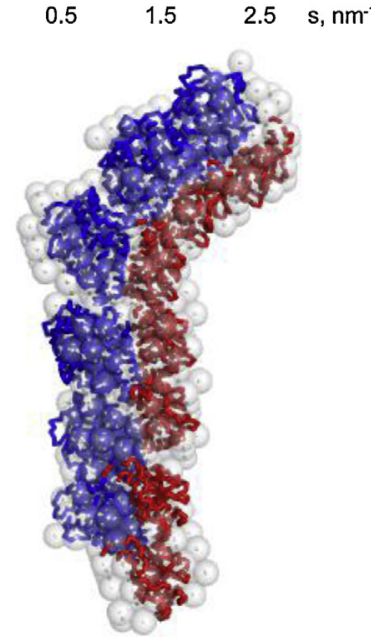

AT3Q24-tubulin complex

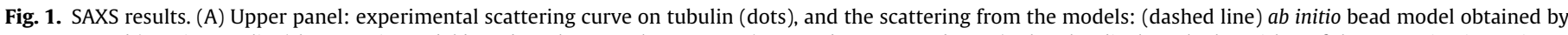

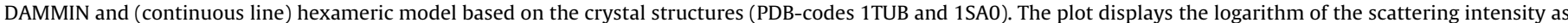

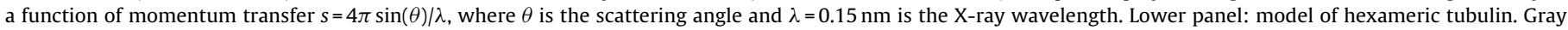

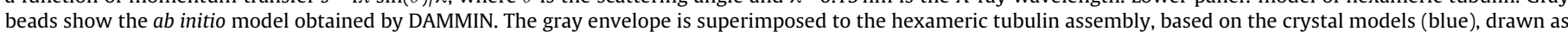

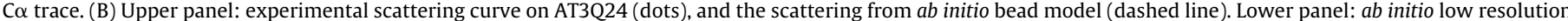

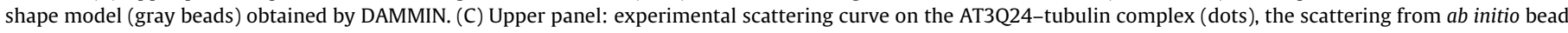

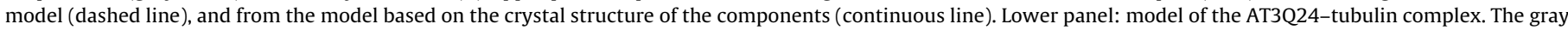

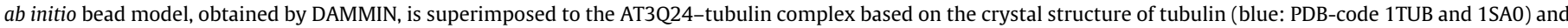

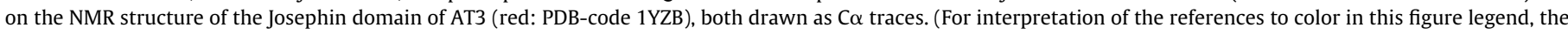
reader is referred to the web version of the article.) 
experimental SAXS curve for the AT3Q24. The estimated molecular weight of the solute $\left(\mathrm{MW}_{\exp }=260 \pm 20 \mathrm{kDa}\right)$ suggests that the AT3 oligomer should have 6-7 monomers under the assayed experimental conditions. Accordingly, the excluded volume of the particle in solution is $V_{p}=425 \pm 20 \mathrm{~nm}^{3}$. The experimental $R_{g}=6.9 \pm 0.3 \mathrm{~nm}$ and maximum size $D_{\max }=25 \pm 1 \mathrm{~nm}$ point to an elongated particle structure. The low resolution shape of AT3Q24, reconstructed $a b$ initio using DAMMIN (Svergun, 1999), has the overall size of about $50 \AA \times 60 \AA \times 240 \AA$, fitting the experimental data with discrepancy $\chi=1.07$ (Fig. 1B, upper panel). Overall, the AT3Q24 oligomer ab initio model is similar in size to that of tubulin, but it has a more bent shape (Fig. 1B, lower panel). A high resolution model of AT3 oligomer cannot be built in the $a b$ initio low resolution envelope, considering that the JD (PDB-code 1YZB) is only half of the total scattering mass of the protein.

The SAXS parameters calculated from measurement on the AT3Q24-tubulin complex are $\mathrm{MW}_{\exp }=500 \pm 30 \mathrm{kDa}$, $V_{p}=900 \pm 30 \mathrm{~nm}^{3}, \quad R_{g}=8.4 \pm 0.4 \mathrm{~nm}$ and maximum size $D_{\max }=30 \pm 2 \mathrm{~nm}$, distinctly different from those of the isolated species. The low resolution shape of the AT3Q24-tubulin complex, reconstructed $a b$ initio using DAMMIN (Svergun, 1999), has the overall size of about $60 \AA \times 80 \AA \times 280 \AA$, fitting the experimental data with discrepancy $\chi=1.11$ (Fig. 1C, upper panel). A high resolution model of the complex was attempted based on the following considerations: (1) the $a b$ initio model shows that the complex is more elongated than the single components (tubulin and AT3Q24), and (2) the radius of the section of the complex is much larger than the radius of the section of the single components, but it is smaller than their summation. Therefore, the scaffold of the complex was modeled by the linear addition of tubulin $\alpha \beta$ dimers with the JD intercalating laterally between tubulin monomers, so as to minimize the increased radius of the section (Fig. 1C, bottom panel). This is in agreement with the expectation that the AT3Q24 oligomer interacts with the tubulin oligomer in a "parallel" fashion, since under physiological conditions the AT3Q24 protein should face and bind the external part of the microtubule. Accordingly, in our model the JD units contact the tubulin surface at the region that would be external to the tubulin protofilament. Given that this model misses the unstructured part of $\mathrm{AT} 3 \mathrm{Q} 24$ and the relative position of tubulin and the JD is not uniquely defined, the computed curve displays some deviations from the experiment at higher angles leading the discrepancy value of $\chi=1.65$ (Fig. 1C, upper panel). Still, the fit at low angles is rather good indicating that the tentative model correctly represents the overall structure of the AT3Q24-tubulin complex.

\subsection{Optimal tubulin binding of AT3Q24 involves co-presence and appropriate spacing of three separate amino acid stretches}

Following the SAXS analysis, we performed SPR experiments to achieve a more precise identification of the AT3 regions involved in tubulin dimer binding. We studied real time association and dissociation using a sensor chip coupled directly to tubulin dimer and assaying different truncated forms of human AT3Q24 (N-terminal His-tagged proteins) (Fig. 2). The binding and release of each variant to and from the chip was monitored. To determine the $K_{D}$ values, we used a Langmuir 1:1 model fitting of simultaneous sensorgrams at different concentrations with BIA evaluation software. No binding kinetics displayed appreciable deviations from such fitting (Fig. S1).

Only for the variants AT3Q24, AT3Q24-3UIM, AT3 ${ }_{1-291}$ and AT3Q6 (constructs 1, 2, 4 and 8) measurable values could be recorded, all other forms displaying binding below the detection limit (Figs. 2, S2, and S3). In particular, we could assign to the AT3Q24 splice variant (construct 1) an affinity as low as $50 \mathrm{nM}$, in keeping with our previous report (Mazzucchelli et al., 2009). The truncated AT3 $1-291$ form (construct 4) was capable of binding tubulin dimer , although with an about 20 -fold lower affinity with respect to the AT3Q24 wild type variant. Surprisingly, the longer construct 3 , in which the polyQ stretch is still present, lost any detectable binding, suggesting that the polyQ region may interfere with tubulin binding in the absence of region 319-362. These data suggest that a tubulin binding region (TBR) is present downstream of the polyQ. We will refer to this site as TBR3. The presence of a binding region downstream of the polyQ was further supported by comparing the affinities of the two AT3 splicing isoforms mainly expressed in the central nervous system (Goto et al., 1997; Schmidt et al., 1998), i.e., AT3Q24 (construct 1) and AT3Q24-3UIM (construct 2), which only differ in the C-terminal region (Figs. 2, S2, and S4).

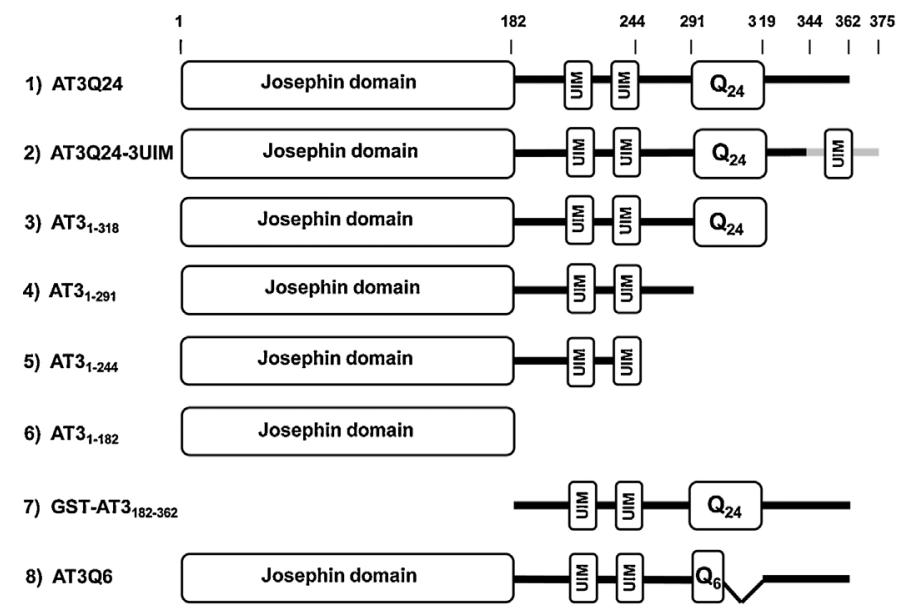

\begin{tabular}{|c|c|c|c|c|c|}
\hline \multicolumn{3}{|c|}{$\alpha \beta$-TUBULIN } & \multicolumn{3}{|c|}{ HDAC6 } \\
\hline $\operatorname{kon}\left(M^{-1} s^{-1}\right)$ & $k_{\text {off }}\left(s^{-1}\right)$ & $K_{D}(M)$ & $k_{o n}\left(M^{-1} s^{-1}\right)$ & $\operatorname{Koff}\left(s^{-1}\right)$ & $K_{D}(M)$ \\
\hline $1.7 \cdot 10^{4}$ & $9.2 \cdot 10^{-4}$ & $5.2 \cdot 10^{-8}$ & $8.7 \cdot 10^{2}$ & $6.3 \cdot 10^{-4}$ & $7.2 \cdot 10^{-7}$ \\
\hline $6.2 \cdot 10^{2}$ & $1.7 \cdot 10^{-3}$ & $2.7 \cdot 10^{-6}$ & $2.9 \cdot 10^{2}$ & $4.1 \cdot 10^{-5}$ & $1.4 \cdot 10^{-7}$ \\
\hline b.d. & b.d. & b.d. & b.d. & b.d. & b.d. \\
\hline $6.9 \cdot 10^{2}$ & $7.2 \cdot 10^{-4}$ & $1.0 \cdot 10^{-6}$ & b.d. & b.d. & b.d. \\
\hline b.d. & b.d. & b.d. & n.a. & n.a. & n.a. \\
\hline b.d. & b.d. & b.d. & b.d. & b.d. & b.d. \\
\hline b.d. & b.d. & b.d. & b.d. & b.d. & b.d. \\
\hline $2.6 \cdot 10^{2}$ & $1.4 \cdot 10^{-3}$ & $5.4 \cdot 10^{-6}$ & n.a. & n.a. & n.a. \\
\hline
\end{tabular}

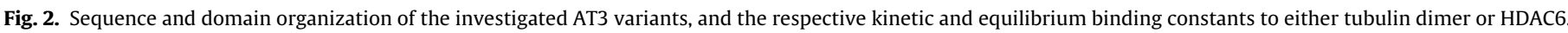

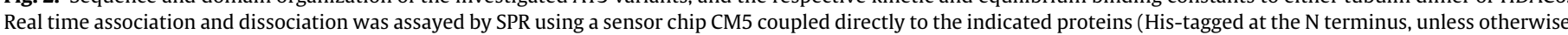

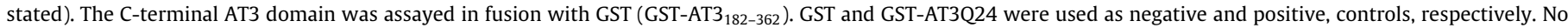

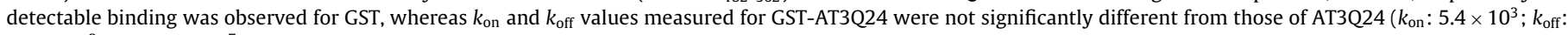
$1.5 \times 10^{-3} ; K_{D}: 2.8 \times 10^{-7}$ ). UIM: ubiquitin-binding motif; b.d.: below detection; n.a.: not assayed. For other details see Section 2 . 
A

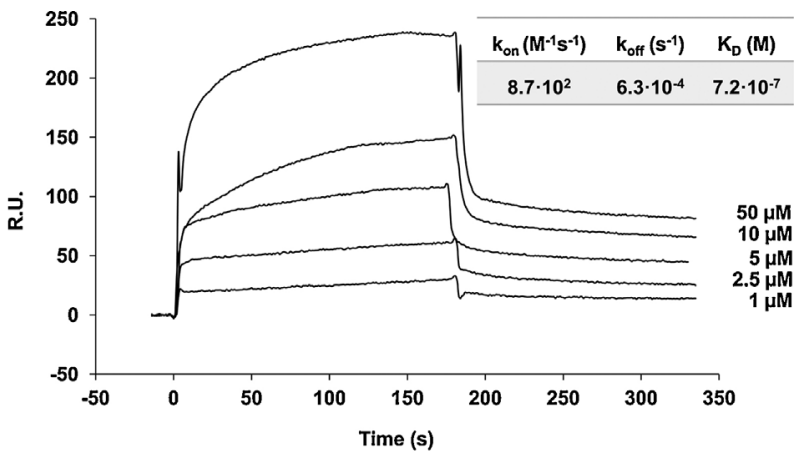

B

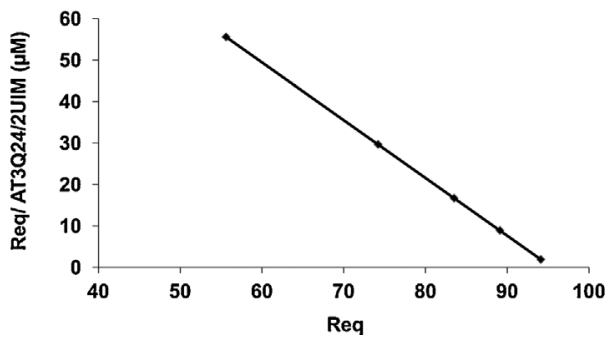

Fig. 3. Association/dissociation kinetics for the binding between HDAC6 and AT3Q24. (A) HDAC6 was immobilized on the sensor chip and the indicated concentrations of AT3Q24 were flowed onto the chip surface. (B) The Req values obtained for each given protein concentration were used to generate the Scatchard plot. In the inset the kinetic and equilibrium binding constants are given.

For the isoform AT3Q24-3UIM, a $2.7 \mu \mathrm{M} K_{D}$ was determined, close to that of the truncated AT3 ${ }_{1-291}$ mutant, suggesting that TBR3 is either absent or functionally inactivated in this splice variant. Also noteworthy is that the AT3Q6 form (construct 8) displayed an affinity well below that of AT3Q24, suggesting that a polyQ length above a given threshold is required for the correct positioning of TBR3.

Further deletion of the disordered region (construct 5) resulted in undetectable binding in the BIAcore assay, suggesting that one binding region upstream of the polyQ is located in the stretch 244-291. We refer to this region as TBR2. Although JD did not show any binding to tubulin in our SPR assay, it should be stressed that our previous pull-down experiments also demonstrated that the JD in isolation was capable of binding tubulin (Mazzucchelli et al., 2009). We therefore assume that its affinity for tubulin dimer is below the sensitivity of the BIAcore $\mathrm{X}$ instrument. Along with the SAXS data reported in this work and the previous pull-down experiments, the essential role of JD in tubulin binding was also confirmed by the lack of any detectable tubulin binding of the C-terminal disordered domain in isolation (construct 6, GST-AT3 $182-362$ ).

On the whole, these results support the idea that AT3 interacts with tubulin dimer via three regions, one located in the JD and the two others in the C-terminal disordered domain, namely in the stretches 244-291 and 319-362, respectively.

\subsection{Ataxin-3 directly binds HDAC6}

A previous report has shown that in the transport machinery of misfolded proteins to the aggresome via microtubules, HDAC6 recognizes protein aggregates by binding to polyubiquitylated proteins at the diglycine motifs of the unanchored ubiquitin C-termini generated by AT3 (Ouyang et al., 2012). However, to date it is unclear whether HDAC6 interacts directly with AT3. We therefore performed further SPR experiments, using a sensor chip coupled to HDAC6, to assess the binding and release of the AT3 variants under investigation. Actual sensorgrams are reported in Fig. 3 and summarized in Fig. 2. Direct binding of AT3 to HDAC6 was substantiated by significant affinity values, in the range $10^{-7}$ to
$10^{-8} \mathrm{M}$ (Figs. 2 and 3). The two AT3Q24 and AT3Q24-3UIM isoforms displayed quite similar affinities (Figs. 2 and S5). However, no significant binding by any of the truncated variants was detected (Fig. S5). The 5-fold difference between the two isoforms suggests that the HDAC6-binding site includes the C-terminal region of AT3 downstream of the polyQ region.

\section{Discussion}

Aggresomes are proteinaceous inclusion bodies that accumulate around the microtubule-organizing center in eukaryotic cells, when cellular degradation machinery is impaired or overwhelmed, leading to an accumulation of protein for disposal (Johnstron et al., 1998). Their formation is regarded as a protective response, sequestering potentially cytotoxic aggregates and also acting as a staging center for eventual autophagic clearance from the cell (Kawaguchi et al., 2003; Olanow et al., 2004; Ross and Poirier, 2005).

Several molecular players are involved in sorting misfolded proteins to aggresomes via microtubules. In particular, polyubiquitin-tagged proteins are gathered by HDAC6, which also binds the component dynactin/p150 Glued of dynein, the microtubule-based motor protein (Kawaguchi et al., 2003; Ouyang et al., 2012). Furthermore, previous work has suggested that the ZnF-UBP domain of HDAC6 binds protein aggregates by interacting with polyubiquitin moieties exclusively at the level of their unanchored C-termini. In addition, AT3 has been implicated as one of the deubiquitinases responsible for exposing such termini on the aggregates, so quite likely it plays a major role in their sorting to the aggresome (Ouyang et al., 2012). A previous report (Rodrigues et al., 2010) also added to the understanding of AT3's physiological role by showing that its depletion causes a significant disorganization of the cytoskeleton, including microtubules, microfilaments and intermediate filaments, in line with our previous finding that AT3 is capable of tightly binding to tubulin (Mazzucchelli et al., 2009). Finally, HDAC6 is also likely to be associated, at least reversibly, with microtubules, given its well-known tubulin deacetylase activity (Valenzuela-Fernández et al., 2008).

These data clearly indicate that the transport machinery of aggregated proteins to aggresomes is a quite complex one, wherein HDAC6 acts as the hub protein, interacting with at least dynein, microtubules and polyubiquitin. Thus, the present investigation was mainly aimed at providing a better understanding of AT3's role in such machinery.

We first characterized the AT3-tubulin dimer complex by SAXS, which actually confirmed the capability of the two protein species to tightly interact with each other. In particular, based on the assumption that under physiological conditions AT3Q24 should face and bind the external part of the microtubule, the data highlight an interaction in which the AT3Q24 oligomer binds to the tubulin oligomer in a "parallel" fashion with the JD units intercalating laterally between tubulin monomers (Fig. 1C, bottom panel).

Consistently with SAXS data, SPR data show that tubulin dimer and AT3 interact according to a Langmuir 1:1 model. Interaction of AT3Q24 with tubulin takes place through a single binding interface, since the secondary plot of $\ln [d(\mathrm{RU}) / d($ time $)]$ against time shows a curve with a constant slope, indicative of a single binding site (Fig. S1). Nevertheless, analysis of SPR data indicates that this interaction surface is tripartite, being originated by three discontinuous individual TBRs. Immunoprecipitation experiments reported elsewhere show that the isolated JD may bind tubulin dimer under different experimental conditions (Mazzucchelli et al., 2009), although no binding is detectable by our SPR assay (construct 6 ). We refer to this tubulin interaction region as TBR1. TBR1 deletion (construct 7) completely abolishes binding in our SPR assay. 
The bipartite interaction surface formed by TBR1 and TBR2 - that is located between JD and PolyQ - has at least 100-fold less affinity for tubulin, compared to the tripartite surface found in AT3Q24 (compare constructs 1 and 4 ). When comparing constructs 4 and 8 with the wild type (construct 1 ), it is apparent that a third TBR (referred to as TBR3) is located downstream of the polyQ region. Notably, the polyQ stretch plays a modulatory role on AT3-tubulin interaction in a way dependent on the type of tubulin-interacting surface present. Actually, comparison of constructs 1 and 8 indicates that an extended polyQ stretch (Q24) promotes AT3-tubulin interaction when the full tripartite structure can be formed. By contrast, in the absence of TBD3, Q24 inhibits binding ability of the bipartite interface formed by TBD1 and TBD2 (constructs 3 and 4). We speculate that in the case of construct 3 , the polyQ might impair the interaction TBD2/tubulin by intervening between the two binding partners, and/or because of the intrinsically higher thermal factor of an unanchored protein terminus, which would also result in less stable interaction with tubulin by the adjacent TBD2.

Although $K_{D}$ s of the various isoforms and mutants analyzed in this paper differ by more than two orders of magnitude, their $k_{\text {off }}$ are remarkably similar - differing at most by a factor of 2 - indicating that the tripartite structure plays a major role in establishing the interaction, and a much less important role in maintaining it. In the absence of TBR3 - and the more so in the absence of both TBR2 and TBR3 - TBR1 has trouble in establishing a productive contact with tubulin dimer. Once contact has been established, dissociation of the tubulin-AT3 complex is mostly governed by TBR1 that remains locked in place with similar efficacy, regardless of the number of TBRs domains flanking it.

As mentioned above, an AT3 carrying a shorter polyQ (AT3Q6, construct 8) displays an affinity well below that of AT3Q24, suggesting that a polyQ length above a given threshold is required for the correct positioning of TBR3. In line with our observations, a recent paper highlights the crucial role of polyQ length in huntingtin function, as both its expansion and its deletion prevents the upstream and downstream regions from interacting with each other (Caron et al., 2013).

Interestingly, AT3Q24-3UIM - construct 2 - displays an about 50 -fold lower affinity for tubulin than AT3Q24. This suggests that the two isoforms may play different physiological roles, the former likely being the one preferentially involved in the interaction with microtubules.

All together these data indicate that formation of the binding interface to tubulin dimer is governed by a complex intramolecular, inter-domain regulatory network similar to the one found in the much larger human Sos protein (Sacco et al., 2012). We also suggest that the earliest interaction is established with the JD, which possibly helps the other TBRs more easily dock the respective binding sites.

Besides a more in-depth characterization of the mode of AT3-tubulin interaction, one major finding of the present paper is the first evidence we provide of a direct interaction of AT3 with HDAC6. Our data also suggest that HDAC6 binds to the C-terminal stretch of AT3 downstream of the polyQ as supported by the fact that its removal resulted in loss of measurable affinity between the two proteins. Given the comparable affinities of the AT3Q24 and AT3Q24-3UIM isoforms for HDAC6, we suggest that the binding site is located in the stretch 319-344, as it retains an identical sequence in the two variants (Fig. S4).

Although much is still to be elucidated regarding the mechanisms by which the individual components of the transport machinery participate in protein sorting to the aggresome, our results pave the way to further studies that should aid in better understanding their roles and mechanisms. This will be accomplished, in particular, by developing AT3 mutants impaired in their ability to bind either HDAC6 or tubulin dimer, and analyzing the impact of these mutations on the intracellular distribution of such proteins, as well as on aggresome formation.

\section{Acknowledgements}

This work has been supported by grants from Regione Lombardia, Italy (Network-Enabled Drug Design) and from Fondazione Cariplo, Italy (progetto Nobel: Network Operativo per la Biomedicina di Eccellenza in Lombardia) and from Fondazione Cariplo (Progetto Nobel).

\section{Appendix A. Supplementary data}

Supplementary data associated with this article can be found, in the online version, at http://dx.doi.org/10.1016/j.biocel.2014. 03.015 .

\section{References}

Burnett B, Li F, Pittman RN. The polyglutamine neurodegenerative protein ataxin-3 binds polyubiquitylated proteins and has ubiquitin protease activity. Hum Mol Genet 2003;12:195-205.

Burnett BG, Pittman RN. The polyglutamine neurodegenerative protein ataxin-3 regulates aggresome formation. Proc Natl Acad Sci USA 2005:102:4330-5.

Caron NS, Desmond CR, Xia J, Truant R. Polyglutamine domain flexibility mediates the proximity between flanking sequences in huntingtin. Proc Natl Acad Sci USA 2013;110:14610-5.

Chai Y, Berke SS, Cohen RE, Paulson HL. Poly-ubiquitin binding by the polyglutamine disease protein ataxin-3 links its normal function to protein surveillance pathways. J Biol Chem 2004;279:3605-11.

Chow MK, Ellisdon AM, Cabrita LD, Bottomley SP. Purification of polyglutamine proteins. Methods Enzymol 2006;413:1-19.

de Forges H, Bouissou A, Perez F. Interplay between microtubule dynamics and intracellular organization. Int J Biochem Cell Biol 2012;44:266-74.

Doss-Pepe EW, Stenroos ES, Johnson WG, Madura K. Ataxin-3 interactions with rad23 and valosin-containing protein and its associations with ubiquitin chains and the proteasome are consistent with a role in ubiquitinmediated proteolysis. Mol Cell Biol 2003;23:6469-83.

Franke D, Svergun DI. DAMMIF, a program for rapid ab-initio shape determination in small-angle scattering. J Appl Crystallogr 2009;42:342-6.

Garcia-Mata R, Bebok Z, Sorscher EJ, Sztul ES. Characterization and dynamics of aggresome formation by a cytosolic GFP-chimera. J Cell Biol 1999;146:1239-54.

Gatchel JR, Zoghbi HY. Diseases of unstable repeat expansion: mechanisms and common principles. Nat Rev Genet 2005;6:743-55.

Goto J, Watanabe M, Ichikawa Y, Yee SB, Ihara N, Endo K, et al. Machado-Joseph disease gene products carrying different carboxyl termini. Neurosci Res 1997;28:373-7.

Harris GM, Dodelzon K, Gong L, Gonzalez-Alegre P, Paulson HL. Splice isoforms of the polyglutamine disease protein ataxin-3 exhibit similar enzymatic yet different aggregation properties. PLoS ONE 2010;5:e13695.

Johnstron JA, Ward CL, Kopito RR. Aggresomes: a cellular response to misfolded proteins. J Cell Biol 1998;143:1883-98.

Kawaguchi Y, Kovacs JJ, McLaurin A, Vance JM, Ito A, Yao TP. The deacetylase HDAC6 regulates aggresome formation and cell viability in response to misfolded protein stress. Cell 2003;115:727-38.

Konarev PV, Volkov VV, Sokolova AV, Koch MHJ, Svergun DI. PRIMUS. A WindowsPC based system for small-angle scattering data analysis. J Appl Crystallogr 2003;36:1277-82

Kozin MB, Svergun DI. Automated matching of high- and low-resolution structural models. J Appl Crystallogr 2001;34:33-41.

Masino L, Musi V, Menon RP, Fusi P, Kelly G, Frenkiel A, et al. Domain architecture of the polyglutamine protein ataxin-3: a globular domain followed by a flexible tail. FEBS Lett 2003;549:21-5.

Mazzucchelli S, De Palma A, Riva M, D’Urzo A, Pozzi C, Pastori V, et al. Proteomic and biochemical analyses unveil tight interaction of ataxin-3 with tubulin. Int J Biochem Cell Biol 2009;41:2485-92.

Natalello A, Frana AM, Relini A, Apicella A, Invernizzi G, Casari C, et al. A major role for side-chain polyglutamine hydrogen bonding in irreversible ataxin-3 aggregation. PLoS ONE 2011;6:e18789.

Nicastro G, Menon RP, Masino L, Knowles PP, McDonald NQ Pastore A. The solution structure of the Josephin domain of ataxin-3: structural determinants for molecular recognition. Proc Natl Acad Sci USA 2005;102:10493-8.

Nogales E, Wolf SG, Downing KH. Structure of the alpha beta tubulin dimer by electron crystallography. Nature 1998;391:199-203.

Nogales E, Whittaker M, Milligan RA, Downing KH. High-resolution model of the microtubule. Cell 1999;96:79-88.

Olanow CW, Perl DP, DeMartino GN, McNaught KS. Lewy-body formation is an aggresome-related process: a hypothesis. Lancet Neurol 2004;3:496-503 [Review]. 
Ouyang H, Ali YO, Ravichandran M, Dong A, Qiu W, Mackenzie F, et al. Protein aggregates are recruited to aggresome by histone deacetylase 6 via unanchored ubiquitin C termini. J Biol Chem 2012;287:2317-27.

Porod G. General theory. In: Glatter O, Kratky O, editors. Small-angle X-ray scattering. London: Academic Press; 1982.

Ravelli RB, Gigant B, Curmi PA, Jourdain I, Lachkar S, Sobel A, et al. Insight into tubulin regulation from a complex with colchicine and a stathmin-like domain. Nature 2004;428:198-202.

Rodrigues AJ, do Carmo Costa M, Silva TL, Ferreira D, Bajanca F, Logarinho E, et al. Absence of ataxin-3 leads to cytoskeletal disorganization and increased cell death. Biochim Biophys Acta 2010;1803:1154-63.

Roessle MW, Klaering R, Ristau U, Robrahn B, Jahn D, Gehrmann T, et al. Upgrade of the small-angle X-ray scattering beamline X33 at the European Molecular Biology Laboratory, Hamburg. J Appl Crystallogr 2007;40; 190-4.

Ross CA, Poirier MA. Opinion: what is the role of protein aggregation in neurodegeneration? Nat Rev Mol Cell Biol 2005;6:891-8.

Sacco E, Farina M, Greco C, Lamperti S, Busti S, Degioia L, et al. Regulation of hSos1 activity is a system-level property generated by its multi-domain structure. Biotechnol Adv 2012;30:154-68.

Schmidt T, Landwehrmeyer GB, Schmitt I, Trottier Y, Auburger G, Laccone F, et al An isoform of ataxin-3 accumulates in the nucleus of neuronal cells in affected brain regions of SCA3 patients. Brain Pathol 1998;8:669-79.
Svergun DI, Barberato C, Koch MHJ. CRYSOL - a program to evaluate X-ray solution scattering of biological macromolecules from atomic coordinates. J Appl Crystallogr 1995;28:768-73.

Svergun DI. Restoring low resolution structure of biological macromolecules from solution scattering using simulated annealing. Biophys J 1999;76: 2879-86.

Valenzuela-Fernández A, Cabrero JR, Serrador JM, Sánchez-Madrid F. HDAC6: a key regulator of cytoskeleton, cell migration and cell-cell interactions. Trends Cell Biol 2008;18:291-7.

Volkov VV, Svergun DI. Uniqueness of ab initio shape determination in small angle scattering. J Appl Crystallogr 2003;36:860-4.

Winborn BJ, Travis SM, Todi SV, Scaglione KM, Xu P, Williams AJ, et al. The deubiquitinating enzyme ataxin-3, a polyglutamine disease protein, edits Lys63 linkages in mixed linkage ubiquitin chains. J Biol Chem 2008;283:26436-43.

Zoghbi HY, Orr HT. Glutamine repeats and neurodegeneration. Annu Rev Neurosci 2000;23:217-47.

Zuccato C, Ciammola A, Rigamonti D, Leavitt BR, Goffredo D, Conti L, et al. Loss of huntingtin-mediated BDNF gene transcription in Huntington's disease. Science 2001;293:493-8

Zuccato C, Tartari M, Crotti A, Goffredo D, Valenza M, Conti L, et al. Huntingtin interacts with REST/NRSF to modulate the transcription of NRSE-controlled neuronal genes. Nat Genet 2003;35:76-83. 